\title{
Effects of intensive blood pressure lowering on the progression of chronic kidney disease: a systematic review and meta-analysis
}

\author{
Jicheng Lv MD PhD, Parya Ehteshami BSc, Mark J. Sarnak MD, Hocine Tighiouart MS, Min Jun PhD, \\ Toshiharu Ninomiya MD PhD, Celine Foote MD, Anthony Rodgers MD PhD, Hong Zhang MD PhD, \\ Haiyan Wang MD, Giovanni F.M. Strippoli MD PhD, Vlado Perkovic MD PhD
}

See related commentary by Hildebrand and Garg on page 941 and at www.cmaj.ca/lookup/doi/10.1503/cmaj.130168

\begin{abstract}
Background: Recent guidelines suggest lowering the target blood pressure for patients with chronic kidney disease, although the strength of evidence for this suggestion has been uncertain. We sought to assess the renal and cardiovascular effects of intensive blood pressure lowering in people with chronic kidney disease.

Methods: We performed a systematic review and meta-analysis of all relevant reports published between 1950 and July 2011 identified in a search of MEDLINE, Embase and the Cochrane Library. We included randomized trials that assigned patients with chronic kidney disease to different target blood pressure levels and reported kidney failure or cardiovascular events. Two reviewers independently identified relevant articles and extracted data.
\end{abstract}

Results: We identified 11 trials providing information on 9287 patients with chronic kidney disease and 1264 kidney failure events (defined as either a composite of doubling of serum creati- nine level and $50 \%$ decline in glomerular filtration rate, or end-stage kidney disease). Compared with standard regimens, a more intensive blood pressure-lowering strategy reduced the risk of the composite outcome (hazard ratio [HR] $0.82,95 \%$ confidence interval $[\mathrm{Cl}] 0.68-0.98$ ) and end-stage kidney disease (HR 0.79, 95\% Cl 0.67$0.93)$. Subgroup analysis showed effect modification by baseline proteinuria $(p=0.006)$ and markers of trial quality. Intensive blood pressure lowering reduced the risk of kidney failure (HR $0.73,95 \% \mathrm{Cl} 0.62-0.86)$, but not in patients without proteinuria at baseline (HR 1.12, 95\% Cl 0.67-1.87). There was no clear effect on the risk of cardiovascular events or death.

Interpretation: Intensive blood pressure lowering appears to provide protection against kidney failure events in patients with chronic kidney disease, particularly among those with proteinuria. More data are required to determine the effects of such a strategy among patients without proteinuria.

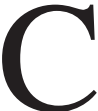
hronic kidney disease is a major public health problem worldwide, affecting $10 \%-15 \%$ of the adult population. ${ }^{1}$ Blood pressure-lowering agents are the mainstay of management strategies aiming to slow the progression of chronic kidney disease, as well as a core aspect of strategies aiming to reduce cardiovascular risk. ${ }^{2-4}$ Observational studies have shown a log-linear increase in the risk of kidney failure with high blood pressure levels across the observed range, ${ }^{5-7}$ suggesting that further lowering blood pressure could reduce the risk of kidney failure at most blood pressure levels. Current guidelines recommend a blood pressure target below 130/80 $\mathrm{mm} \mathrm{Hg}$ for patients with chronic kidney disease, ${ }^{8-10}$ but this recommendation is mostly based on observational studies and a single randomized trial (the Modification of Diet in Renal Disease [MDRD] study) that focused on kidney protection. ${ }^{11}$ Subsequent trials of different targets in people with chronic kidney disease have yielded inconsistent results, ${ }^{12,13,14}$ leading to criticism by the recent Canadian Hypertension Education Program guideline (which suggested a less aggressive target) of other guidelines, with suggestions that their blood pressure recommendations went beyond the available evidence. This criticism has been supported by a recent systematic review (no meta-analysis was performed) that focused on 3 trials and reported inconclusive results overall but raised the possibility that proteinuria was an effect modifier. ${ }^{15}$ The final result has been clinician uncertainty about optimal blood pressure levels in patients with chronic kidney disease.
Competing interests: Jicheng Lv has received grant support from Pfizer for hypertension research. Anthony Rodgers has received an unrestricted grant from Dr. Reddy's Laboratories for a trial that includes blood pressurelowering agents. Vlado

Perkovic has received honoraria from Servier for scientific presentations relating to blood pressure. No other competing interests were declared.

This article has been peer reviewed.

Correspondence to: Vlado Perkovic, vperkovic @george.org.au

CMAJ 2013. DOI:10.1503 /cmaj.121468 
We sought to synthesize the results of all available trials that evaluated the effects of different blood pressure targets in people with chronic kidney disease and to better define the balance of risks and benefits associated with different intensities of blood pressure lowering in this population.

\section{Methods}

\section{Literature search}

We performed a systematic review using the approach recommended in the PRISMA (Preferred Reporting Items for Systematic Reviews and Meta-Analyses) statement. ${ }^{16}$ We identified relevant studies by searching MEDLINE via Ovid (from inception through July 2011), Embase (from inception through July 2011) and the Cochrane Library database (Cochrane Central Register of Controlled Trials, no date restriction) for relevant text and medical subject headings that included all spellings of "antihypertensive agents," "target blood pressure," "intensive blood pressure treatment," "strict blood pressure treatment" and "tight blood pressure control" (Appendix 1, available at www.cmaj.ca/lookup/suppl/doi :10.1503/cmaj.121468/-/DC1). We limited our search to randomized controlled trials (RCTs) with at least 6 months' follow-up, but without age or language restrictions. We manually scanned reference lists from identified trials and review articles to identify any other relevant studies. In addition, we searched the ClinicalTrials.gov website for randomized trials that were registered as completed but not yet published.

The literature search, data extraction and quality assessment were done independently by 2 authors using a standardized approach (JL and PE). All completed RCTs that compared moreversus less-intensive blood pressure targets using pharmacologic blood pressure-lowering agents were eligible for inclusion. Data were extracted either from studies solely involving people with chronic kidney disease or from subsets of other trials where data on patients with chronic kidney disease could be obtained. Chronic kidney disease was determined using the definition developed by the Kidney Disease Outcomes Quality Initiative. ${ }^{8}$

\section{Data extraction and quality assessment}

We obtained published reports for each trial and extracted standard information to a spreadsheet. The data we sought included baseline patient characteristics (age, sex, systolic and diastolic blood pressure, history of diabetes, history of hypertension and chronic kidney disease), blood pressure targets in each arm of the study, types of blood pressure-lowering agents used, length of follow-up, change in systolic and diastolic blood pressure during the trial, outcome events and adverse events. We assessed the risk of bias using the approach recommended by the Cochrane Collaboration (assessing allocation concealment; blinding of patients, investigators and outcome assessors; completeness; intentionto-treat analysis). For 2 studies, we requested additional information from the original authors by written correspondence, which we included in our analysis. ${ }^{12,14}$ Any differences in data extraction were resolved by discussion among the authors and, where required, adjudication by a third reviewer (VP).

\section{Outcomes}

Our primary outcome was a composite of 50\% decline in glomerular filtration rate and doubling of the serum creatinine level, or end-stage kidney disease. We also collected data on major cardiovascular events (i.e., fatal and nonfatal myocardial infarction, fatal and nonfatal stroke) and allcause mortality, where available.

\section{Data synthesis and analysis}

We calculated individual study hazard ratios (HRs) with 95\% confidence intervals (CIs) ${ }^{13,14,17,18}$ (we extracted relative risks [RRs] when HRs were not available) for each outcome before pooling. For the continuous measurement of blood pressure, we used the mean difference between groups. We obtained summary estimates using a random effects DerSimonian-Laird model. We looked for evidence of heterogeneity in treatment effect between patients with and without baseline proteinuria by comparing summary results obtained from relevant subgroups (protein-creatinine ratio $>0.22$ or daily protein excretion $>300 \mathrm{mg}$ ) in a prespecified subanalysis. We estimated the percentage of variability across studies attributable to heterogeneity beyond chance using the $I$ statistic..$^{19}$ We made graphic representations of potential publication bias using Begg funnel plots of the natural logarithm of the HR versus its standard error and assessed them visually. ${ }^{20} \mathrm{~A} 2$-sided $p$ value less than 0.05 was considered statistically significant.

\section{Results}

\section{Search results and characteristics of included studies}

Our literature search returned 2524 results yielding 1650 potentially relevant articles. Of these articles, we reviewed the full text of 67 reports, from which we identified 12 publications relating to 11 relevant RCTs (Figure 1). These trials 
involved a total of 9287 participants with chronic kidney disease (Table 1). ${ }^{13,14,17,1,21-28}$ Because kidney failure typically develops slowly over time, we included the long-term post-trial follow-up data from the African American Study of Kidney Disease and Hypertension (AASK; 8.8-12.2-yr follow-up) ${ }^{13}$ and MDRD trials (16.7-yr followup), although the blood pressure differences between the 2 arms were not significant during the cohort phase in these 2 studies. The reported trial quality varied substantially. All of the trials used open designs, although patients were blinded in 2 trials. Four of the 11 trials did not describe allocation concealment, ${ }^{17,18,21,28}$ and another 3 did not describe whether the analysis was done by intention-to-treat ${ }^{23-25}$ (Appendix 2, available at www.cmaj.ca/lookup/suppl/doi:10 .1503/cmaj.121468/-/DC1)
Blood pressure targets varied substantially between trials. Two trials targeted mean blood pressure levels below $92 \mathrm{~mm} \mathrm{Hg}$ in the intensive treatment arm, with a target of $107 \mathrm{~mm} \mathrm{Hg}$ in the standard treatment arm. ${ }^{17,26}$ One trial aimed for a blood pressure target below 130/80 $\mathrm{mm} \mathrm{Hg}$ compared with a diastolic blood pressure of $90 \mathrm{~mm} \mathrm{Hg},{ }^{14} 1$ study targeted below 120/ $80 \mathrm{~mm} \mathrm{Hg}$ as compared with 135-140/85$90 \mathrm{~mm} \mathrm{Hg},{ }^{28}$ and 4 studies had diastolic blood pressure targets below $75-80 \mathrm{~mm} \mathrm{Hg}$ with comparators ranging from 80 to $90 \mathrm{~mm} \mathrm{Hg}{ }^{.1,23-25} \mathrm{~A}$ trial involving pediatric patients targeted a 24hour mean blood pressure below the 50th percentile, compared with the 50th to 95th percentiles in the control group. ${ }^{18}$ Two trials had more liberal targets for intensive treatment $(<140$ $150 \mathrm{~mm} \mathrm{Hg}$ systolic and $85 \mathrm{~mm} \mathrm{Hg}$ diastolic). ${ }^{22,29}$

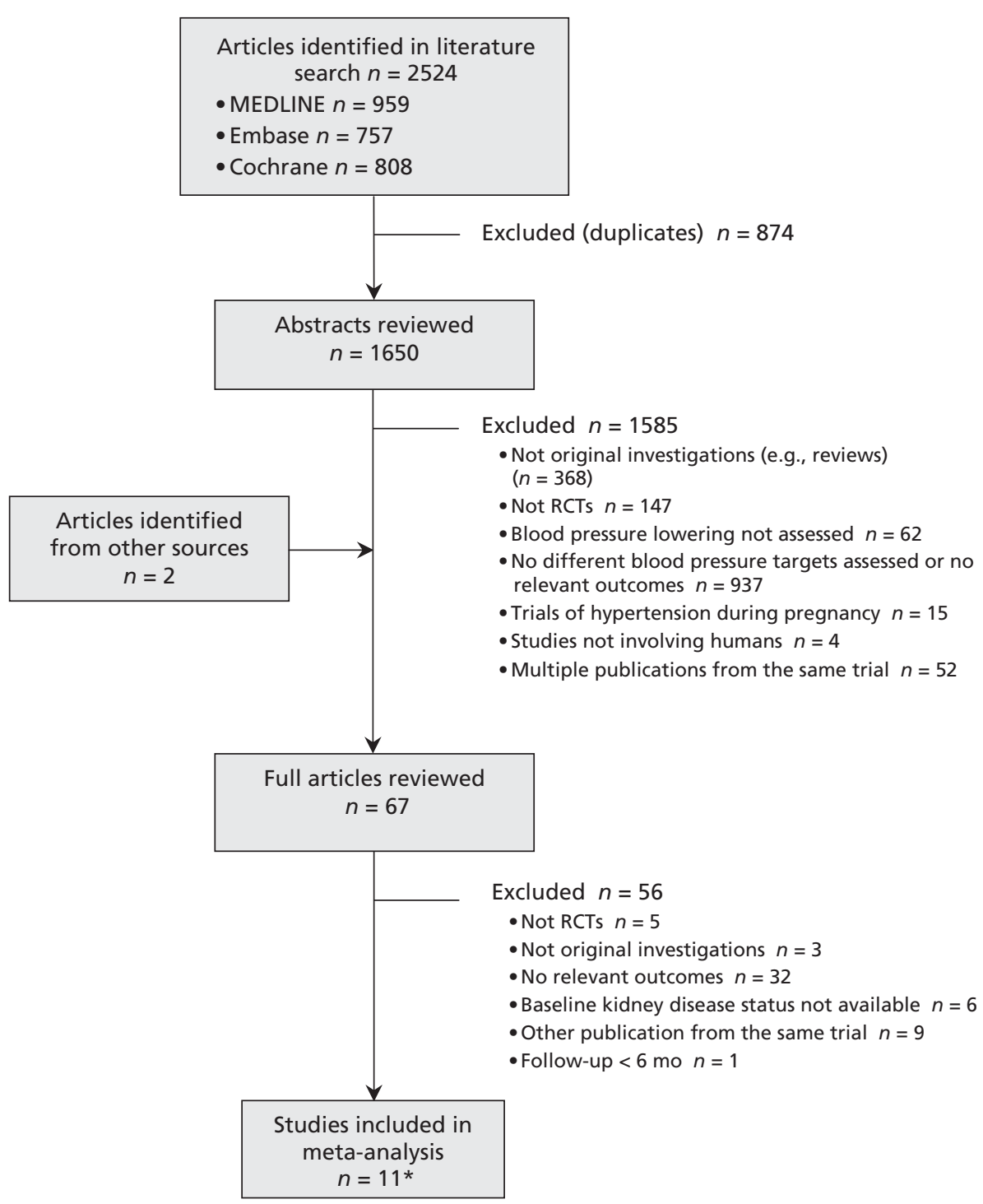

Figure 1: Identification of relevant studies for inclusion in the meta-analysis. RCT = randomized controlled trial. *Included studies were published in 12 articles. 


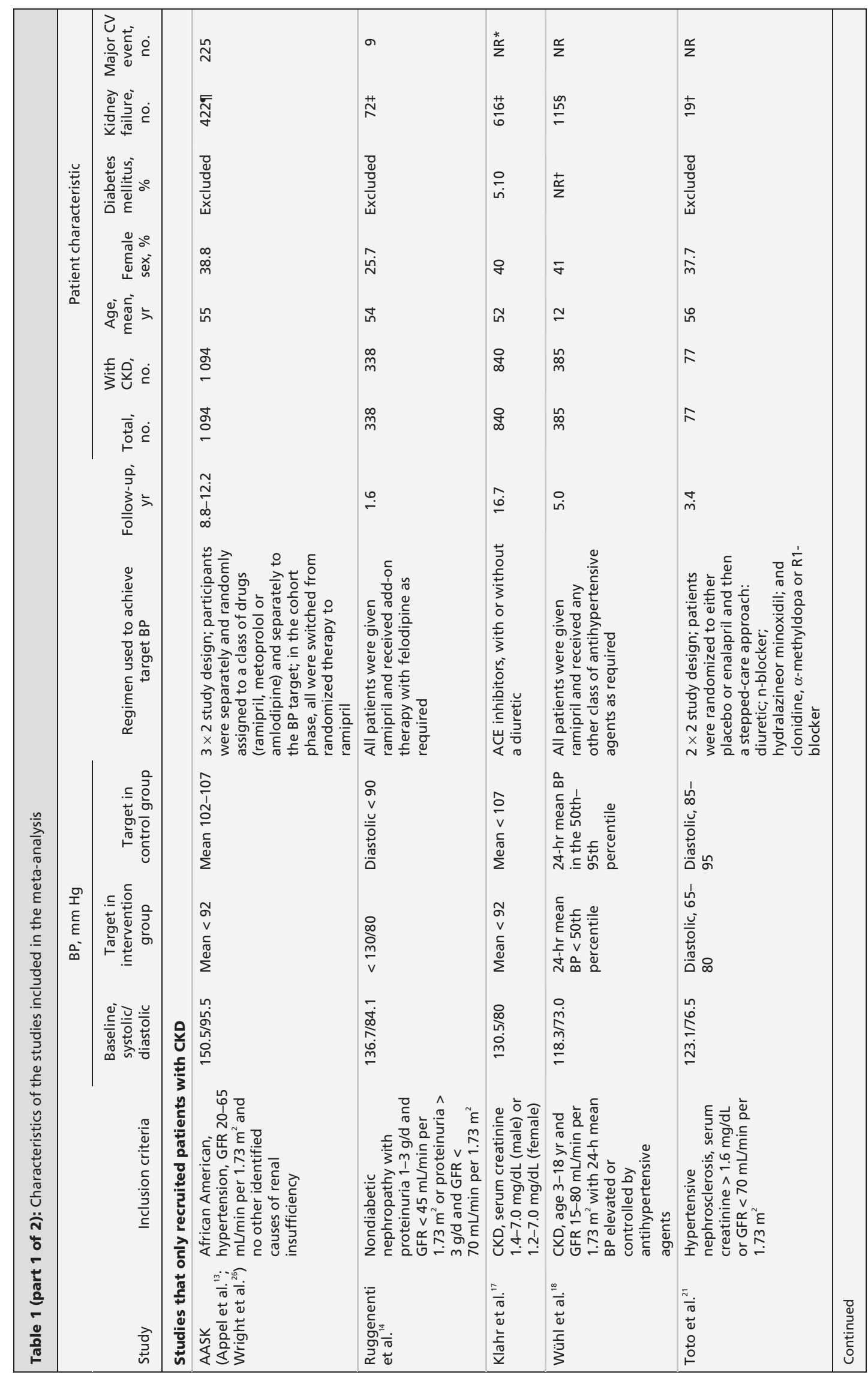




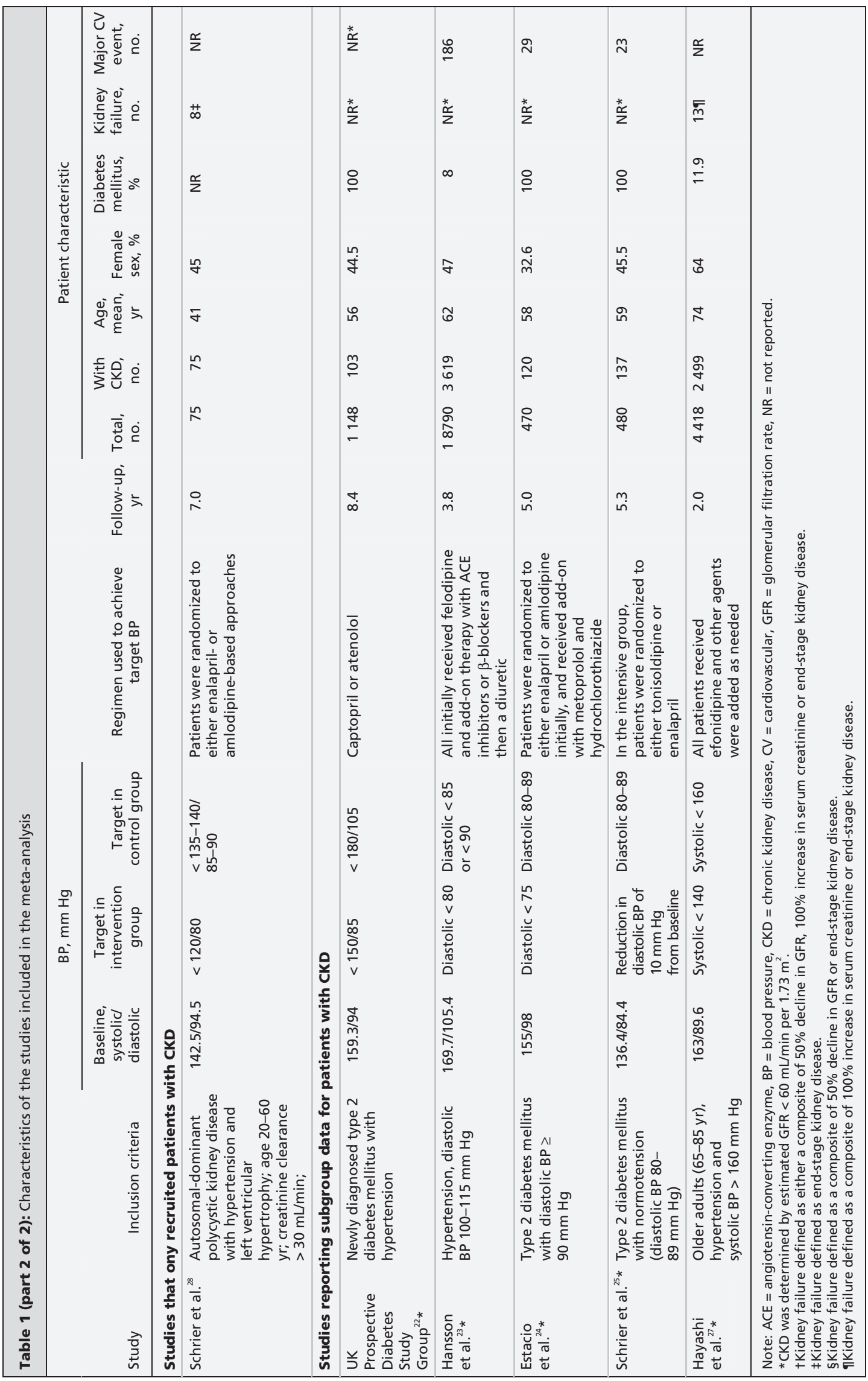




\section{Effects of intensive blood pressure lowering on renal and cardiovascular outcomes}

\section{Renal outcomes}

Seven trials involving 5308 participants recorded a total of 1264 kidney failure events. $14,17,18,21,26-28$ A -7.7-mm Hg difference in systolic blood pressure and a $-4.9-\mathrm{mm} \mathrm{Hg}$ difference in diastolic blood pressurem was seen between the 2 treatment arms in the studies that reported these differences., ${ }^{4,17,21,26-28}$ Overall, a more intensive regimen reduced the risk of composite kidney failure events by $17 \%$ (HR $0.82,95 \%$ CI $0.68-0.98$ ), and reduced the risk of end-stage kidney disease alone by $18 \%$ (pooled HR for composite outcomes $0.79,95 \%$ CI $0.67-0.93$ ) (Figure 2).

Formal testing did not identify evidence of publication bias, but the number of included trials was small (Appendix 3, available at www.cmaj.ca /lookup/suppl/doi:10.1503/cmaj.121468/-/DC1). We did see mild heterogeneity $\left(I^{2}=38.1 \%, p=0.1\right)$.

Further analysis showed that intensive blood pressure lowering had no effect on kidney failure in patients who did not have proteinuria ( 3 trials involving 1218 patients [HR 1.12, 95\% CI $0.67-$ $1.87]),{ }^{13,17,18}$ but it did reduce the risk of progressive kidney failure by $27 \%$ ( 5 trials involving 1703 patients [HR $0.73,95 \%$ CI $0.62-$ $0.86])^{13,14,17,18,27}$ in people who did have proteinuria at baseline (Figure 3). Subgroup analysis showed heterogeneity between the effect of intensive blood pressure lowering in patients with and without proteinuria ( $p$ for heterogeneity $=0.006$ ). Excluding the study involving pediatric patients ${ }^{18}$ did not change the overall results, with intensive blood pressure lowering reducing the risk of kidney failure in patients with proteinuria (RR 0.76 ,

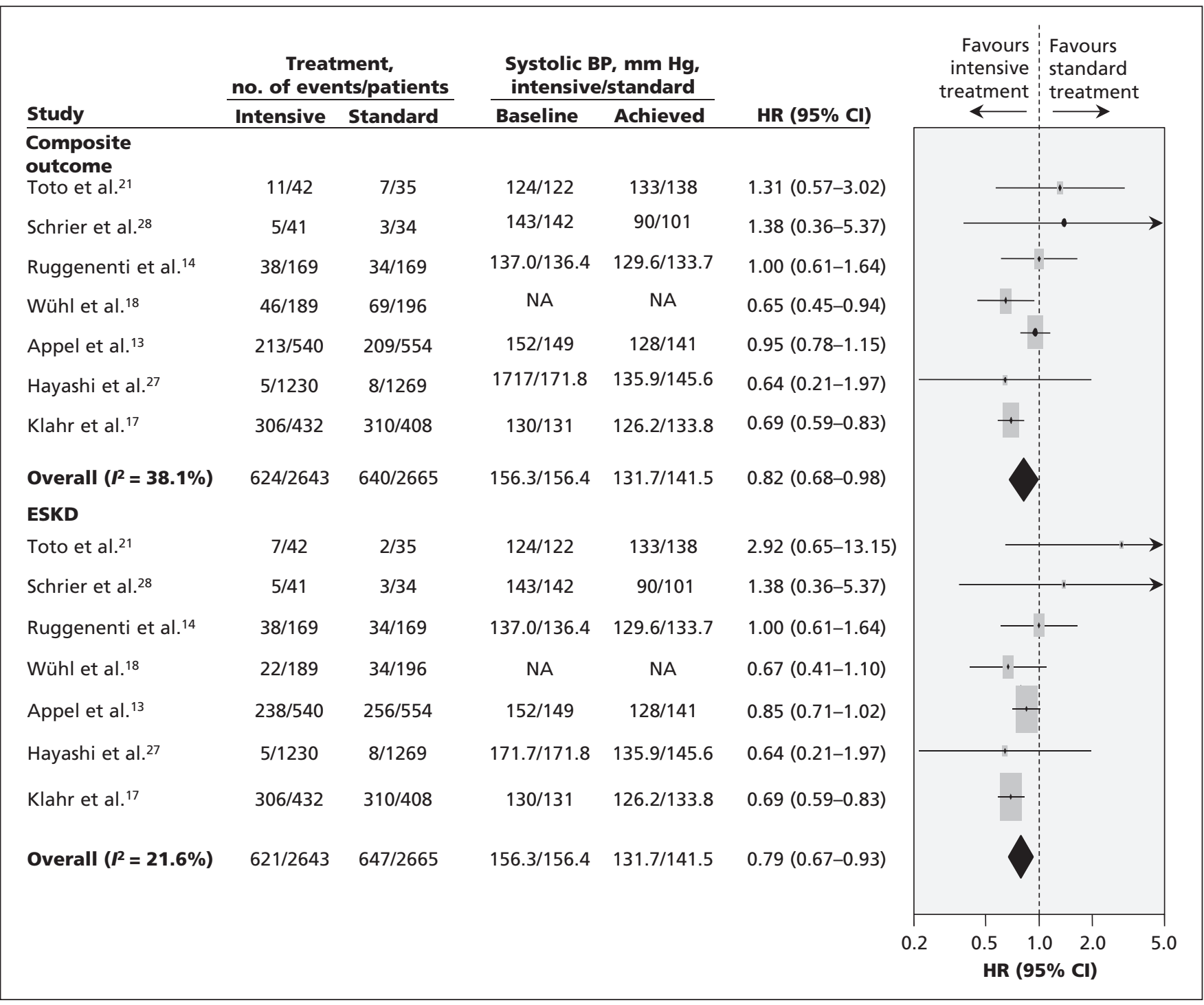

Figure 2: Effects of intensive blood pressure lowering on progressive kidney failure. $\mathrm{BP}=$ blood pressure, $\mathrm{Cl}=\mathrm{confidence}$ interval, ESKD = end-stage kidney disease, HR = hazard ratio. Note: Weights are from random effects analysis. 
95\% CI 0.64-0.89) but not in those without proteinuria (RR 1.03, 95\% CI 0.83-1.25) ( $p$ value for subgroup heterogeneity $=0.03$ ). In addition, we noticed a larger effect was seen in trials where allocation concealment and blinding of outcome assessors were not clearly described ( $p$ for heterogeneity $=0.02$ and 0.04 , respectively) (Appendix 4, available at www.cmaj.ca/lookup/suppl/doi :10.1503/cmaj.121468/-/DC1).

\section{Cardiovascular outcomes}

Major cardiovascular events were reported in 5 trials (472 cardiovascular events in 5308 patients with chronic kidney disease)..$^{13,1423-25}$ Intensive blood pressure lowering did not reduce the risk of cardiovascular events in patients with chronic kidney disease, but the CIs remained wide (RR 1.09, 95\% CI 0.831.42). Six trials reported stroke outcomes (197 events in 5411 patients), ${ }^{13,14,22-25} 5$ trials reported myocardial infarction (138 events in 4317 patients), ${ }^{14,22-25}$ and 5 trials reported heart failure (118 events in 5308 patients). ${ }^{13,14,23-25}$ We saw no clear effect of intensive treatment on any of these vascular outcomes (Appendix 5, available at www.cmaj.ca /lookup/suppl/doi:10.1503/cmaj.121468/-/DC1).

\section{Death}

Ten trials involving 6788 participants reported 846 deaths. ${ }^{13,14,17,18,21-25,28}$ There was no clear effect of intensive blood pressure lowering on risk of all-cause death (RR 0.94, 95\% CI 0.84-1.05) or cardiovascular death (RR 1.20, 95\% CI 0.821.75) (Appendix 5).

\section{Potential harms of treatment}

Two trials reported data on severe adverse events (177 events in 723 participants), and no effect on the risk of such events was seen with intensive blood pressure lowering (RR 1.04, 95\% CI 0.601.78). ${ }^{14,18}$ Three trials reported the risk of hypotension or associated symptoms. ${ }^{17,18,26}$ Individual trials reported that more intensive blood pressure lowering did not increase the risk of hypotension; however, the data were not suitable for metaanalysis. The risk of acute kidney injury was not reported. Finally, there was no clear difference detected in the rate of stopping treatment between the more- and less-intensive treatment groups in the 3 trials that reported this outcome. . $^{14,17,18}$

\section{Interpretation}

Most guidelines suggest that blood pressure should be lowered to below 130/80 $\mathrm{mm} \mathrm{Hg}$ in all patients with chronic kidney disease. ${ }^{8,9,30}$ Our meta-analysis of 11 trials involving more than 9000 participants, among whom more than 1200 kidney failure events were recorded, has shown

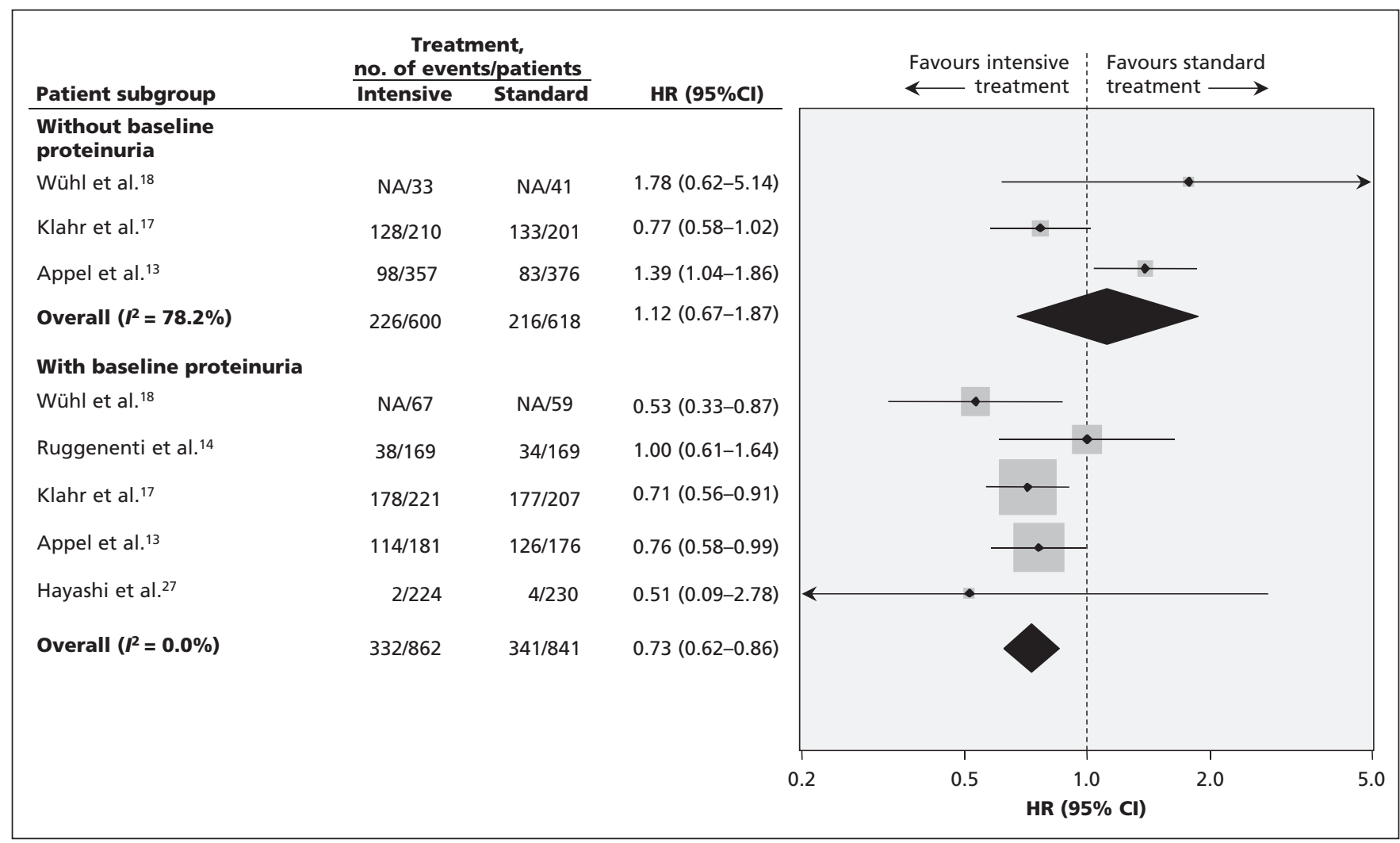

Figure 3: Subgroup analysis of the effect of intensive blood pressure lowering on kidney failure in patients with proteinuria compared with those without proteinuria. $\mathrm{Cl}=$ confidence interval, $\mathrm{HR}=$ hazard ratio. 
that intensive blood pressure control with a lower target reduced the risk of kidney failure. However, the heterogeneity we saw based on markers of study quality raises the possibility that this benefit is overestimated.

Clear heterogeneity can be seen in the results of trials according to the baseline proteinuria levels of participants. Intensive blood pressure lowering provided clear and consistent protection against kidney failure in patients with proteinuria, but not in patients without proteinuria. This trend suggests that aggressive targets may prevent kidney failure in people with proteinuria but not in people with normal urinary protein excretion, highlighting the importance of assessing urinary protein excretion in people with chronic kidney disease.

Our results suggest that a $10 \mathrm{~mm} \mathrm{Hg}$ reduction in systolic blood pressure might result in an overall reduction of $22 \%$ in the risk of kidney failure. This result is consistent with data from observational studies in which lowering systolic blood pressure by $10 \mathrm{~mm} \mathrm{Hg}$ was associated with a lower risk of kidney failure. ${ }^{31-33}$

The results from individual trials were inconsistent, even among trials with the same target blood pressure, highlighting the value of metaanalysis in improving precision and providing an opportunity to identify important risk modifiers with increased statistical power. ${ }^{12,13,17,26}$ Our results suggest clinical heterogeneity in the study population may explain the variable results across these trials, because proteinuria was found to be a clear effect modifier.

The pooled effects of intensive blood pressure lowering on renal outcomes were consistent among studies and subgroups, including participants with proteinuria, with no evidence of heterogeneity. These results were consistent with those from the individual studies that reported an interaction between blood pressure lowering and proteinuria. ${ }^{11,13}$ The mechanisms underlying this observation are unclear, but patients with proteinuria could have high intraglomerular pressure and may therefore be particularly sensitive to intensive blood pressure lowering.

\section{Strengths and limitations}

A key strength of our review is its inclusion of long-term follow-up data from a number of trials with large numbers of end-stage kidney disease events, thus providing substantial statistical power to detect plausible benefits. Kidney function generally declines relatively slowly, highlighting the importance of long-term follow-up to obtain adequate power to show clinically important benefits. Our meta-analysis includes 2 trials involving patients at high risk for end-stage kidney disease with more than 10 years of follow-up, ${ }^{17,34}$ in addi- tion to the ESCAPE study with 5 years of followup. ${ }^{18}$ It will be critically important for any new trials assessing interventions aiming to prevent endstage kidney disease to include participants at high risk for this outcome and to follow them for an appropriate period of time to clearly define the presence or absence of any benefits.

A limitation of our study is that most trials of different blood pressure-lowering intensities that evaluated renal outcomes did not include people with diabetic kidney disease. Thus, we cannot answer the question of whether intensive blood pressure lowering is similarly beneficial in that population. The Action to Control Cardiovascular Risk in Diabetes trial investigated the effects of intensive blood pressure control targeting normal systolic pressure (i.e., $<120 \mathrm{~mm} \mathrm{Hg}$ ) in more than 4000 patients with diabetes..$^{35}$ Overall, there was no effect on end-stage kidney disease in the group receiving intensive treatment, as compared with the conventional systolic blood pressure goal of less than $140 \mathrm{~mm} \mathrm{Hg}$. However, this trial has not yet reported the effects of intensive blood pressure lowering according to levels of proteinuria, although these data would be of great interest and value.

Our review was also limited by the varied quality of the included trials and the inability to use a double-blinded study design for a trial of blood pressure targets, raising the risk of differential use of other treatments. In addition, the heterogeneity we saw according to markers of study quality limits the strength of the conclusions that can be drawn. Furthermore, the number of included trials is small, which limited subgroup analysis, and the various definitions used for blood pressure targets made meaningful subgroup analyses by different target levels impossible. Finally, because adverse events were inconsistently reported, safety remains an area of uncertainty.

We did not find any evidence of benefit from intensive blood pressure lowering on cardiovascular events or death. Cardiovascular outcomes were not reported in all of the trials, and the total numbers of events identified were modest, suggesting that this may be an issue of power. Reviews of different blood pressure-lowering targets in the general population have suggested that overall cardiovascular benefit is achieved with lower blood pressure targets ${ }^{36}$ and have refuted concerns derived from observational studies that lower targets in the commonly observed range may lead to increases in the risk of coronary artery disease.

\section{Conclusions}

The available data suggest that intensive blood pressure lowering prevents end-stage kidney disease in patients with chronic kidney disease, particularly in the presence of proteinuria. However, 
the effects remain to be shown in people with normal urinary protein excretion. Our data provide support for current guidelines that suggest lowering blood pressure targets in people with proteinuric chronic kidney disease and highlight the potential value of this approach. More data are required to determine the effect of such a strategy in patients who do not have proteinuria.

\section{References}

1. James MT, Hemmelgarn BR, Tonelli M. Early recognition and prevention of chronic kidney disease. Lancet 2010;375:1296-309.

2. Heerspink HJ, Ninomiya T, Perkovic V, et al. Effects of a fixed combination of perindopril and indapamide in patients with type 2 diabetes and chronic kidney disease. Eur Heart J 2010;31: 2888-96.

3. Ruilope LM, Salvetti A, Jamerson K, et al. Renal function and intensive lowering of blood pressure in hypertensive participants of the hypertension optimal treatment (HOT) study. J Am Soc Nephrol 2001;12:218-25.

4. Jafar TH, Stark PC, Schmid CH, et al. Progression of chronic kidney disease: the role of blood pressure control, proteinuria, and angiotensin-converting enzyme inhibition: a patient-level meta-analysis. Ann Intern Med 2003;139:244-52.

5. Klag MJ, Whelton PK, Randall BL, et al. Blood pressure and end-stage renal disease in men. N Engl J Med 1996;334:13-8.

6. Tozawa M, Iseki K, Iseki C, et al. Blood pressure predicts risk of developing end-stage renal disease in men and women. Hypertension 2003;41:1341-5.

7. Perkovic V, Huxley R, Wu Y, et al. The burden of blood pressure-related disease: a neglected priority for global health Hypertension 2007;50:991-7.

8. K/DOQI clinical practice guidelines for chronic kidney disease evaluation, classification, and stratification. Am J Kidney Dis 2002;39(Suppl 1):S1-266.

9. Chobanian AV, Bakris GL, Black HR, et al. The seventh report of the Joint National Committee on Prevention, Detection, Evaluation, and Treatment of High Blood Pressure: the JNC 7 report. JAMA 2003;289:2560-72.

10. Mancia G, Laurent S, Agabiti-Rosei E, et al. Reappraisal of European guidelines on hypertension management: a European Society of Hypertension Task Force document. J Hypertens 2009;27:2121-58.

11. Peterson JC, Adler S, Burkart JM, et al. Blood pressure control, proteinuria, and the progression of renal disease. The Modification of Diet in Renal Disease study. Ann Intern Med 1995;123:754-62.

12. Sarnak MJ, Greene T, Wang X, et al. The effect of a lower target blood pressure on the progression of kidney disease: long-term follow-up of the modification of diet in renal disease study. Ann Intern Med 2005;142:342-51.

13. Appel LJ, Wright JT Jr, Greene T, et al. Intensive blood-pressure control in hypertensive chronic kidney disease. $N$ Engl J Med 2010;363:918-29.

14. Ruggenenti P, Perna A, Loriga G, et al. Blood-pressure control for renoprotection in patients with non-diabetic chronic renal disease (REIN-2): multicentre, randomised controlled trial. Lancet 2005;365:939-46

15. Upadhyay A, Earley A, Haynes SM, et al. Systematic review: blood pressure target in chronic kidney disease and proteinuria as an effect modifier. Ann Intern Med 2011;154:541-8.

16. Liberati A, Altman DG, Tetzlaff J, et al. The PRISMA statement for reporting systematic reviews and meta-analyses of studies that evaluate health care interventions: explanation and elaboration. Ann Intern Med 2009;151:W65-94.

17. Klahr S, Levey AS, Beck GJ, et al. The effects of dietary protein restriction and blood-pressure control on the progression of chronic renal disease. Modification of Diet in Renal Disease Study Group. N Engl J Med 1994;330:877-84.

18. Wühl E, Trivelli A, Picca S, et al. Strict blood-pressure control and progression of renal failure in children. N Engl J Med 2009; 361:1639-50.

19. Woodard M. Epidemiology: design and data analysis. 2nd ed. Boca Raton (FL): Chapman \& Hall/CRC Press; 2005;687-90.

20. Egger M, Davey Smith G, Schneider M, et al. Bias in metaanalysis detected by a simple, graphical test. BMJ 1997;315: 629-34.

21. Toto RD, Mitchell HC, Smith RD, et al. "Strict" blood pressure control and progression of renal disease in hypertensive nephrosclerosis. Kidney Int 1995;48:851-9.
22. Tight blood pressure control and risk of macrovascular and microvascular complications in type 2 diabetes: UKPDS 38. UK Prospective Diabetes Study Group [published erratum in $B M J$ 1999;318:29]. BMJ 1998;317:703-13.

23. Hansson L, Zanchetti A, Carruthers SG, et al. Effects of intensive blood-pressure lowering and low-dose aspirin in patients with hypertension: principal results of the Hypertension Optimal Treatment (HOT) randomised trial. HOT Study Group. Lancet 1998;351:1755-62.

24. Estacio RO, Jeffers BW, Gifford N, et al. Effect of blood pressure control on diabetic microvascular complications in patients with hypertension and type 2 diabetes. Diabetes Care 2000;23 (Suppl 2):B54-64.

25. Schrier RW, Estacio RO, Esler A, et al. Effects of aggressive blood pressure control in normotensive type 2 diabetic patients on albuminuria, retinopathy and strokes. Kidney Int 2002;61:1086-97.

26. Wright JT Jr, Bakris G, Greene T, et al. Effect of blood pressure lowering and antihypertensive drug class on progression of hypertensive kidney disease: results from the AASK trial. JAMA 2002;288:2421-31.

27. Hayashi K, Saruta T, Goto Y, et al. Impact of renal function on cardiovascular events in elderly hypertensive patients treated with efonidipine. Hypertens Res 2010;33:1211-20.

28. Schrier R, McFann K, Johnson A, et al. Cardiac and renal effects of standard versus rigorous blood pressure control in autosomaldominant polycystic kidney disease: results of a seven-year prospective randomized study. J Am Soc Nephrol 2002;13:1733-9.

29. JATOS Study Group. Principal results of the Japanese trial to assess optimal systolic blood pressure in elderly hypertensive patients (JATOS). Hypertens Res 2008;31:2115-27.

30. Mancia G, De Backer G, Dominiczak A, et al. 2007 Guidelines for the Management of Arterial Hypertension: The Task Force for the Management of Arterial Hypertension of the European Society of Hypertension (ESH) and of the European Society of Cardiology (ESC). J Hypertens 2007;25:1105-87.

31. Lewington S. Blood pressure, cholesterol and premature death: towards the real relationship. Oxford (UK): University of Oxford; 1999.

32. Klag MJ WP, Randall BL, Neaton JD, et al. Blood pressure and end-stage renal disease in men. N Engl J Med 1996;334:13-8.

33. O'Seaghdha CM PV, Lam TH, McGinn S, et al.; Asia Pacific Cohort Studies Collaboration. Blood pressure is a major risk factor for renal death: an analysis of 560352 participants from the Asia-Pacific region. Hypertension 2009;54:509-15.

34. Appel LJ, Anderson CA. Compelling evidence for public health action to reduce salt intake. $N$ Engl J Med 2010;362:650-2.

35. Gerstein HC, Miller ME, Byington RP, et al. Effects of intensive glucose lowering in type 2 diabetes. N Engl J Med 2008;358: 2545-59.

36. Lv J, Neal B, Ehteshami P, et al. Effects of intensive blood pressure lowering on cardiovascular and renal outcomes: a systematic review and meta-analysis. PLoS Med 2012;9:e1001293.

Affiliations: The George Institute for Global Health (Lv, Ehteshami, Jun, Foote, Rodgers, Perkovic), The University of Sydney, Sydney, Australia; Renal Division, Department of medicine (Lv, Zhang, Wang), Peking University First Hospital, Beijing, China; Tufts Medical Center (Sarnak, Tighiouart), Boston, Mass.; the Department of Medicine and Clinical Science Graduate School of Medical Sciences (Ninomiya), Kyushu University, Japan; the Centre for Kidney Research (Strippoli), The Children's Hospital at Westmead, School of Public Health, The University of Sydney, Sydney, Australia; the Department of Pharmacology and Epidemiology (Strippoli), Mario Negri Sud Consortium, S Maria Imbaro, Chieti, Italy; and DIAVERUM Medical-Scientific Office (Strippoli), Lund, Sweden

Contributors: Vlado Perkovic, Jicheng Lv and Parya Ehteshami conceived the idea for the study. Jicheng Lv, Parya Ehteshami, Mark J. Sarnak, Hocine Tighiouart and Vlado Perkovic collected, analyzed and interpreted the data. Jicheng Lv, Parya Ehteshami, Vlado Perkovic drafted the manuscript. All of the authors revised the manuscript for important intellectual content and approved the final version submitted for publication.

Funding: Jicheng Lv was supported by an Amgen Renal Research Fellowship. Vlado Perkovic was supported by an Australian Heart Foundation Career Development Award. The funders had no role in study design, data collection and analysis, decision to publish, or preparation of the manuscript. 